\title{
Sachregister zu Band 85
}

Die fett gedruckten Zahlen bedeuten Eigenarbeiten. Bb. = Buchbesprechungen.

Ablatio retinae $174,184 \bullet-$ sympathische 175

- $\quad$ Netzhautspaltung mit einerÖffnung 108

postoperative - nach Zyklo-dialyse 5

operativ geheilte - bei Nystagmus und Aphakie 360

- Elektrolyse gegen - -336

- Kontaktschale als Schutzglas

bei Operation der - 186 - zur Geschichte der Katholyse

der - 2 Adaptation 232 Afokale Systeme, halbstarre 181 Agranulozytose, Erkrankung d.

Anhangsgebilde der Augen auf

dem Boden der - 117 Akkommodation 343 (Bericht) Albinotiker, Lichtschutzschalen

für - 186 Amblyopie, einseitige vorüber-

gehende 184 Anatomie 153 (Bericht) Aneurysmen, intrakranielle 51 Angioide Netzhautstreifen 62 ,

253 Angiomatosis retinae 253 Angiome der Lider, Behandlung

der - mit sklerosierenden Ein-

spritzungen 61 Anhangsgebilde, Erkrankung

der - auf dem Boden der Agranulozytose 117 Aniridie 139, 244 Ankyloblepharon 144, 243

Anomaloskop 230 Anophthalmus congenitus 136 Anthropologie 148 (Bericht) Aphakie, operativ geheilte Netz-

hautablösung bei - 360 Arachnodaktylie 358 Argolaval-Augensalbe 361 Arsenobenzol,

Sehnervenerkran-

kung nach 184 Arteria centralis retinae, Em-

bolie der - 213 Arteria hyaloidea 140 Astigmatismus, Entstehung des

einseitigen -181

- $\quad$ inverser 182

- $\quad$ Hornhaut-A. und Total-A. 264!- und Myopie 247

Azetylcholin 345 Augenhintergrundininfrarotem Licht 265

weiß punktierter 143

seltene Befunde am -184 Augenkliniken, zur Vorgeschich-

te der Wiener - 326 Augenlider s. Lider Augenmuskellähmungen, an-

geborene 144 Augenmuskeln, erbliche Affek-

tionen der - 244 Augenspiegel mit Rotfilter 268 Augenverletzungen, gewerb-

liche 185

B

Bindehaut s. Conjunctiva Binokulares Sehen 235 Blendungsschmerz 221 Blindheit und Eugenik 255 - optokinetischer Nystagmus bei

hysterischer $-65,172$ Bluttransfusion bei schweren

Sehstörungen nach Blutverlust 
31 Blutverlust, Bluttransfusion bei

schweren Sehstörungen nach ·

31 Botulismus 266 Brille, Geschichte der - 152 Bulbusfistel, hintere 163

$\mathrm{C}$

Calcarinagegend, Veränderun-gen der -42

- $\quad$ Schädigungen der - bei Ek-lampsie 160

Carabelli-Sabouraudscher Höcker bei kongenitaler Syphilis des Auges 189

Chiasma 39

Cholesteatome der Orbita 146

Chorioidea, Knochenbildung in der -217

Conjunctiva, Anatomie der -

154

Gitterfasern in der $\mathbf{-}-24$

- Anomalien der - 138, 243 - Veränderung der - bei lym-phatischer Leukämie 165

- $\quad$ Tuberkulöser Primäraffekt ander - mit Erythema nodosum270

$24^{*}$

370 Sachregister zu Band 85.

Conjunctiva, Behandlung der Verätzungen der - 166

- $\quad$ Bindehautplastik bei der Elliot-schen Operation 73

Cornea, Anatomie der - 155

Entwicklung der -132

erbliche Anomalien der - 248

familiäre Degeneration der -

347

Verdünnung der - - bei guter Sehschärfe 186

Randatrophie der - nach Schä-delbruch 60

Ringabszeß der -61

Nekrose der -186

Keratozele bei Dystrophia mar-ginalis der $\cdot-82$

Corneatrübung, angeborene 139

$\mathrm{D}$

Dakryozystitis acuta 180 Dehnsucht des Auges 1 Delirium tremens, Skotome bei

- 37Dermoidzysten der Orbita 146Descemetokele persistens 186Descemetsche Membran, Auf-

rollung der - nach Zyklodialyse 185 Diabetes und Auge 166

- $\quad$ Refraktionsanomalien bei - 339Dichloräthylsulfid, Wirkung

des auf das Auge 355 Druck, intraokularer, Hervor-rufung einer Steigerung des - 265

- $\quad$ Stauungspapille bei Senkung des

$-\quad 266$

- $\quad$ Binnendruck des entzündetenAuges 180

Ductus-naso-lacrimalis 149 Dunkeladaptation 232 Dunkelzitternkleiner Kinder 121

Dynamometer nach Sobanski 165

E Eidetische Fähigkeiten 238 Eigenbluteinspritzung in die Vorderkammer bei tuberkulöser Iridozyklitis 16 Ektopia lentis, angeborene 244 Ektropium, Lupusnarben-E. 176 Elektrolyse gegen Netzhautab- 
lösung 336 Elliotsche Operation, Binde-haut- und Mundschleimhaut-plastik bei der - 73

Embolie der Arteria centralis retinae 213 Embryontoxon 249 Endophlebitistuberculosa164

Entoptische Wahrnehmungen 224

Entoptoskop 225

Entwicklungsgeschichte des Auges 132 (Bericht)

Enzephalitis epidemica, Au-generscheinungen nach - 48

Epikanthus, Vererbbarkeit des - 144

Epilepsie, Netzhautveränderun-gen bei - 53

Epitheldystrophie, familiäre juvenile - der Hornhaut 249

Erbkrankheiten des Auges 350

Erythema nodosum, tuberkulöser Primäraffekt an der Conjunctiva mit - 270

Eugenik und Blindheit 255

Euryblepharon 144

Exkavation, kesself örmige - der Papule ohne Tensionserhöhung 185

Exophthalmus, pulsierender 176

$\mathrm{F}$

Farben, Wirkung von 239 Farbenblindheit 250 Farbensinn iinîi. Fettembolie der Netzhaut 59

Formwahrnehmungen 239

G

Gefäßbildung im Glaskörper 186

Geflügellähme 161

Gehirnabszeß, Augensymptome bei - 52

Gehirnblutungen, Augensymptome bei - 51

Gehirnentwicklung, Einflußder

- $\quad$ auf den Gesichtsschädel 154Gehirnsklerose, Netzhauttumo-

ren bei - 52, 253 Gehirntumoren 49 Geschichte der Ophthalmologie

149 (Bericht) Gesichtsfeld 231 Gestaltswahrnehmungen 239 Gewerbliche Augenverletzun-

gen 185 Gitterfasern in der Bindehaut 24 Glaskörper, Anatomie des - 159

Entwicklung des - 133

Gefäßbildung im - 186 Glaukom 135, 245, 261

schweres juveniles -184

- $\quad$ Beziehungen des - zum Gefäß-system 180

Glioma retinae, Vererbung des

- 246Gonorrhoische Komplement-

bindungsreaktion 183 Größenwahrnehmungen 239

Sachregister zu Band $85 . \quad 371$

$\mathrm{H}$

Hamburgsche Behandlung bei

tabischer Sehnervenatrophie 196 Haptik der gebogenen Kontakt-

schalen 180 Haut, ererbte Anomalien der -

63 (Bsp.) Hemeralopie 251 Hemianopsie, Ursachen bilempo-

raler - bei Schwangerschaft 88 - bei Hypophysiserkrankungen

39. $4^{\circ}$ Herpes corneae und Trauma 353 Heterochromia iridis 140 Hippelsche Netzhauterkrankung mit operativ geheilter

Kleinhimzyste 352 van der Hoeve series Syndrom

249 Hornhaut s. Cornea Hydrophthalmus congenitus 
I Ichthyosis congenita mit Êk-

tropion 244 Idiotie, familiar e amaurotische

$251 \cdot-$ Refraktion und Linsenbeschaf-

fenheit bei - 342 Interorbitalbreite, Variabilität

der - 243 Iridektomie, sackförmige Hernie

des Pigmentblattes nach peri-

pherer - 261 Iridenkleisis antiglaucoma-

tosa, Ophthalmia sympathica

nach - 268 Iridozyklitis, Eigenbluteinsprit-

zung in die Vorderkammer bei

tuberkulöser - 16 Iris, Anomalien der - 140, 249 •-Abreißung der - bei nicht per-

forierender Verletzung 82

$\mathrm{J}$

Jahreszeiten, Einfluß der - auf die Entstehung von Augen-krankheiten 58

$\mathrm{K}$

Kammerdränage bei Entzün-dungen der vorderen Augenteile 267

Kapselpinzette für Starextrak-tion 187

Katarakt 158, 249, 250

Katarakt. Stammbaum mit sel-

tener Häufung von ·-310 Kataraktoperation 174

- $\quad$ Kapselpinzette für intrakapsu-läre - 187

Katholyse der Netzhautablösung,

Geschichte der -2 Keratitis disciformis 347 Keratitis parenchymatosa

186, 188 Keratoconjunctivitis sicca,

Allgemeinsymptomatologie bei

- $\quad$ 266Keratokonus 248Kerato-Mykose 181Keratozele bei Dystrophia mar-

ginalis corneae 82 Kleinhimzyste bei Hippelscher

Netzhauterkrankung 352 Kniehöcker, Erkrankungen der

- $\quad$ 4iKnochenbildung in der Ader-

haut 217 Knochenplatte im Auge 174 Kolobome 138, 143, 244 -- Entstehung der atypischen 189

- $\quad$ angeborene - der Oberlider 243-匹 Bildung von - - bei totalem

Pupillenverschluß durch Nach-starschwarte 57

Komplementbindungsreak-tion, gonorrhoische 183

Konstitution und Tuberkulose 268

Kontaktschalen, Haptik der gebogenen - -180

- $\quad$ als Schutzglas bei Operation derAblatio retinae 186

Kopfverletzungen, Augensym-

ptome bei - 51 Krukenbergsche Pigment-

spindel 245 Kryptophthalmus, partieller

J43

$\mathrm{L}$

Leukämie, Bindehaut- und Trä-

nensackveränderung bei lym- 
phatischer - 165 Leukozytenzahl und Tuberku-

loseantigen AO. 314 Lichtschutzschalen für Albino-

tiker 186 Lichtsinn 223ff. Lider, Anatomie der - 153 a-m erbliche Anomalien der - 243

- $\quad$ angeborene Kürze der .- 144•- Lidspaltenrichtung 149, 243

- $\quad$ Ersatz eines verbrannten Ober-lidrandes 176

372

Sachregister

zu Band 85.

Lidgeschwulst, zystische 354 Lidsperrer, stumpier Doppel-

haken als - 184 Linksglaukosan 180 Linse, Anatomie der - 158 - Anomalien der - - 141, 249

- Doppelbrechung der - 161Linsenluxation, erbliche 244Linsenmangel, angeborener

137Linsennähte, Entwicklung der -

134 Lippenfrischplastik 176 Lipodermoid der Hornhaut 143 Lipoidose 252

Lupusnarben-Ektropium 176 Lymphosarkomatosis, Augen-

veränderungen bei -183

$\mathrm{M}$

Makula, Regenbogenfarbenreflex in der Gegend der - 164

- $\quad$ Bedeutung der - bei der Be-urteilung der Stauungspapille288

Malariabehandlung bei tabi-scher Sehnervenatrophie 44-

Marfanscher Symptomenkom-plex, Vererbung des - 245

Megalokornea 139, 244, 248

Meibom-Mykose 181

Mikrophthalmus 136, 137

Mißbildungen 134 (Bericht)

Mitbewegungen 53

Mongolismus 146

Mundschleimhautplastik bei der Elliotschen Operation 73

Muskelatrophie, progressive 52

Myopie 246, $339 \mathrm{ff}$.

-- Entstehung der - 177

- $\quad$ hochgradige - bei eineiigenZwillingen 352

$\mathrm{N}$

Nachbilder 226

Nachstarschwarte, Kolobom-bildung bei totalem Pupillen-verschluß durch - 57

Nasennebenhöhlenentzün-dungen als Ursache der Neuritis retrobulbaris 36

Nervus oculomotorius, infek-tiöse Lähmung des - 48

Nervus opticus 36 (Bericht)

- Anatomie des - 160

-. Anomalien des $\cdot 250$

- $\quad$ Scheidenblutung des -262 Nervus opticusatrophie, tabi-

sche 44

- Hamburgsche Behandlung beitabischer - 196

Netzhaut s. Retina

Neuritiden, , unbekannter" Ursache 187

Neuritis retrobulbaris 36,178 
Nystagmus, optokinetischer bei hysterischer Blindheit 65, 172

-m operativ geheilte Netzhautab-lösung bei -360

0

Ophthalmia electrica, Netz-

hautveränderungen bei - 172 Ophthalmia sympathica 157 - nach Iridencleisis antiglaucomatosa $268 \bullet-$ intravenöse Salizylbehandlung

der - 360 Ophthalmoenzephalomyelitis

48 Ophthalmologie, Geschichte der

- 149 (Bericht)Ophthalmomalazie 183Optik, physiologische und psycho-

logische -- 223 (Bericht) Orbitaltumoren 161 - Pseudotumor 61 Orbitopalpebralzysten 136

$\mathrm{P}$

Pagetsche Krankheit 253 Panophthalmie, metastatische -

nach septischem Abort 183 Papillitis, enzephalomyelitische

- $\quad$ mit Iritis 357Parotitis und Uveitis auf tuber-

kulöser Grundlage 363 Pathologische Anatomie 153

(Bericht) Pemphigus, geheilter 187 Perimeterbeleuchtung 185 Periphlebitis tuberculosa 164

Pfeifen mit den Tränenwegen 27 Physiologische Optik 223 (Ber.) Pigmentblatt, sackförmige

Her-

nie des - nach peripherer Iri-

dektomie 261 Pigmentierung, Anomalien der

- 245

Poliomyelitis, Augenerscheinun-

gen bei - 49 Prothese, Verletzung durch - 58 Pseudobulbärparalyse, ange-

borene 52 Pseudogliome 364 Pseudostauungspapille 143 Pseudoxanthoma elasticum mit

Gefäßstreifen an der Netzhaut 62, 253 Psychologische Optik 238 (Ber.) Ptosis, Vererbbarkeit der

- 144 Pupillarmembran 140

Sachregister

zu Band 85.

373

Pupillarsaum, glasige Erhebun-gen am - bei Rubeosis iridis diabetica 262

Pupillenstöru ngen bei Nerven-syphilis 45

Pupillotonie 52

E

Raumsinn 233 Raumwahrnehmungen 239 Reflexbild an der hinteren Lin-

senoberfläche 264 Refraktion 182, 246, 338 (Bericht) Regenbogenf arbenreflexinder

Maculagegend 164 Retina, Anatomie der - 159

Anomalien der - $-\mathbf{1 4 3}, 250$

Altersentartung des Pigment-epithels der - 163

endokrine Beziehungen der Pig-mentdegeneration der - 181

- Fettembolie der - 59

- Veränderungen der - bei Ophthalmia electrica 172

- Gefäßstreifen an der - bei Pseudoxanthoma elasticum 62,

253 Retinitis circinata, Behandlung

der - 365 Retinitis pigmentosa $252-$ bei multipler Sklerose 46 Retinitis proliferans 174

Retinoschisis 108 Röntgenlicht, Sehen im ·- 224 Rotfilter, Verbesserung des Seh-vermögens bei 
getrübten Augen-medien mit $\mathbf{\square -} 268$ Rubeosis iridis diabetica, glasige Erhebungen am Papillarsaum bei - 262

S Salizylbehandlung, intravenöse - der sympathischen Ophthal-

mie 360 Schädelbruch, Randatrophie der

Hornhaut nach ·- 60 Schädelmessungen 148 Schielen, Ein- und Aufwärts-Sch.

170 Schleimzyste, angeborene - - des

Unterlides 137 Schwangerschaft, Ursachen bi-

temporaler Hemianopsie bei ·-

88 Schwindelerscheinungen 63 Sehbahn 36 (Bericht), 160 Sehpurpur 226 Sehschärfe 233, 342

(Bericht) Sellabrücken 188

Sinus cavernosus, Thrombophlebitis des -161

Ski era, erbliche Anomalien der - 249

Skleralsporn, Anatomie des -

157 Sklerose, multiple, Augenstö-

rungen bei - 45Íf- - als Ursache von Neuritiden 187

- $\quad$ Erklärung des zentralen Sko-toms bei - 351

Skotom, zentrales - bei multipler

Sklerose 351 Sporotrichose des unteren Trä-

nenkanälchens 181 Status dysraphicus 358 Stauungspapille 38, 49 •-ロ Entstehung der - 362, 363

bei intraokularer Drucksenkung 266

am teilweise atrophischen Seh-nerven 352

Bedeutung des blinden Fleckes bei der Beurteilung der - 288

Synästhesie 238 Syphilis und Auge 43, 44

- $\quad$ der Carabelli-SabouraudscheHöcker bei angeborener -· desAuges 189

T Tebeprotininjektionen, sub-konjunktivale - zur Diagnostik und Behandlung tuberkulöser

Augenerkrankungen 271 Tiefenwahrnehmung 233 Tiere, optische Beobachtungen an

- 241Tonsillenerkrankungen, interne Komplikationen der entzünd-lichen - 270

Toyparsamid 44

Trachom 154, 177, 274, 348, 368

(Bb.) Tränenbein, Größe und Form des

- $\quad$ 149Tränenkanälchen, Sporotrichose des unteren - 181

Tränenorgane, Anatomie der -

157 Tränensack, Veränderung des -

bei lymphatischer Leukämie 165 Tränensackfistel, angeborene

144 Tränenwege, Pfeifen mit den 27 Tuberkelbazillen, Züchtung der

- - aus dem strömenden Blut 269 Tuberkulinbehandlung, perku $<$ •

tane - mit Hilfe von Glas- oder

Schmirgelpapierabreibung 191 Tuberkulose und Konstitution

268

374 Namenregister zu Band 85.

Tuberkulose, Diagnostik und Behandlung tub. Augenerkran-kungen mittels subkonjunkti-valer

Tebeprotininjektionen 271

- tub. Primäraffekt an der Conjunctiva mit Erythema nodosum 270

Tuberkuloseantigen A.O 354

- und das Verhalten der Leuko-zytenzahl 314

Turmschädel 145, 254 
$\ddot{\mathrm{u}}$

Überhitzungdes Körpers, Augen-

symptome bei - 161 Uvea, Anatomie der - 157 Uveitis und Parotitis auf tuber-

kulöser Grundlage 363

Velonoskiaskopie 263, 300 Ventrikulographie, Sen- und

Gesichtsfeldstörungen nach

43

Verätzung durch den Saft der

Wolfsmilchpflanze 173 Vererbungund Auge 243 (Bericht) Vorderkammerpunktion bei

narbiger Verkürzung der Über-

gangsfalte 180

W

Wetter, Einfluß des - auf die

Entstehung von Augenkrank-

heiten 58 Wiener Augenkliniken, zurVor-

geschichte der - 326 Wolfsmilchpflanze, Verätzung

des Auges durch den Saft der -

173

$\mathrm{Z}$

Zilioretinale Gefäße 141 Zwillingsuntersuchungen 254 Zyklodialyse, Aufrollung der

Descemet nach - 185 - postoperative Netzhautablösung

nach $\square-5$ Zyklopie 136

Namenregister zu Band 85.

Abramson 45 Accardi 232 Adelmann 136 Adler 342

Adrogue 143, 229, 230 Agnello 243 Aiakawa 149 Ajtai 182 Alaerts 235 Alajmo 246 Albers 145

Albrich 179 Allen 229, 230 Aoyama 149 Arjona 239 Aszalós 185 Aubineau 189 Aust 349

B

Bader 150 Bahn 152 Balado 42 Bamber 140 Barcia Goyanes 42 Bard 261 Bardanzeller 148

Barnes 49 Barry 228

Bartels i45, i59, i70ff. Barth 224

Bartley 235 Beisbarth 142 Bell 256 Bendenritter 184,

186 Bender 137, 154, 240 Beniuc 241 Berg 150, 224, 264 Berliner 268 Bernays 229 Bertondi

225 Best 246 Beyermann 52 Beyne 234 Bianchi 132 Bickel 353 Biemond 51 Birch-Hirschfeld

233 Biro 144, 18i; 244 Blatt 36 Blondel 225 Böck 162 Bodechtel 160 Boehm 161 van Bogaert

244 Bokermann 173 Bonnet 231 Bonvec 247 Boros 187 Bourdier 229 Bousfield 228

ter Braak 48, 51 Brana 178, 187 Brand 223 Bresgen 217 Brockmeyer 121 Bucciante 254

Buchnell 135 Bucklers 350, 354,362 Buddenbrock 223 Buhss 61 Bunge 249 Busacca 158

C

C. Caramazza 39 Chase 226 Clark 225 Cocchi 140 Cockayne 63 Cohrs 143 Colenbrander 228

Colrat 244 v. Conta 63 Cook 233 Coppez 53, 145 Coronat 228 Cosmettatos 144 Creed 226 Cruz

136 Crvaric 245 v. Csapody 177 